\title{
SUPER TABLEAUX AND A BRANCHING RULE FOR THE GENERAL LINEAR LIE SUPERALGEBRA
}

\author{
SEAN CLARK, YUNG-NING PENG, AND S. KUANG THAMRONGPAIROJ
}

\begin{abstract}
In this note, we formulate and prove a branching rule for simple polynomial modules of the Lie superalgebra $\mathfrak{g l}(m \mid n)$. Our branching rules depend on the conjugacy class of the Borel subalgebra. A Gelfand-Tsetlin basis of a polynomial module associated to each Borel subalgebra is obtained in terms of generalized semistandard tableaux.
\end{abstract}

\section{INTRODUCTION}

Let $\mathfrak{g l}(m)$ be the general linear Lie algebra and consider the subalgebras

$$
\mathfrak{g l}(1) \subset \mathfrak{g l}(2) \subset \ldots \subset \mathfrak{g l}(m),
$$

where we embed $\mathfrak{g l}(k) \subset \mathfrak{g l}(k+1)$ as the collection of matrices whose last row and column are zero. Then given a finite-dimensional simple $\mathfrak{g l}(m)$-module $L_{m}(\lambda)$, we may restrict the action to view $L_{m}(\lambda)$ as a $\mathfrak{g l}(k)$-module and decompose it into simple $\mathfrak{g l}(k)$-modules $L_{k}(\mu)$; when $k=m-1$, this decomposition is called a branching rule. When $k=1$, this decomposition describes a linear basis for $L_{m}(\lambda)$, which is called the Gelfand-Tsetlin basis ([GW, Chap. 8]). There are several generalizations and applications of the GelfandTsetlin basis ([Mo]). Moreover, this basis has an innate combinatorial description in terms of semistandard Young tableaux, and hence can be used to derive some combinatorial identities.

In this note, we generalize these concepts to the general linear Lie superalgebra $\mathfrak{g l}(m \mid n)$. It is known that there is a branching rule for the standard Borel subalgebra of uppertriangular matrices $([\mathrm{BR}])$. Using Howe duality ([CW2, Se1]), we formulate and prove a branching law with respect to an arbitrary choice of Borel subalgebra $\mathfrak{b}$, and in particular our approach provides a novel proof for the standard Borel case. We then deduce the existence of a Gelfand-Tsetlin basis parametrized by certain tableaux, which we call "bsemistandard". This provides a representation-theoretic proof that the number of these tableaux is independent of the choice of $\mathfrak{b}([\mathrm{Kw}])$.

Acknowledgement. We are grateful to Weiqiang Wang for his suggestion for this collaboration, and for the guidance and advice he has given over the course of this project. We would also like to thank Shun-Jen Cheng for his many helpful conversations. The third author was supported by an REU under Wang's NSF grant.

\section{Preliminaries}

In this note, the underlying field is always $\mathbb{C}$, the complex numbers.

Key words and phrases. Lie superalgebras, representations, Young diagrams, Gelfand-Tsetlin bases, duality. 
2.1. Partitions, Young Diagrams, and Tableaux. Let $\lambda$ be a partition of $n$; that is, a sequence $\lambda=\left(\lambda_{1}, \lambda_{2}, \ldots\right)$ of non-negative integers with $\lambda_{i} \geq \lambda_{i+1}$ and $|\lambda|=\sum_{i} \lambda_{i}=n$. The length of $\lambda$, denoted by $l(\lambda)$, is defined to be the number of non-zero entries of $\lambda$. Each partition corresponds to a unique Young diagram, and we will freely identify a partition with its corresponding Young diagram. We denote the conjugate partition to $\lambda$ by $\lambda^{\prime}$.

A partition $\lambda$ is called an $(m \mid n)$-hook partition if $\lambda_{m+1} \leq n$. We denote the collection of $(m \mid n)$-hook partitions by $P_{m \mid n}$.

Given partitions $\lambda$ and $\mu$ such that $\lambda_{i} \geq \mu_{i}$, let $\lambda / \mu$ denote the skew Young diagram. We will say that a skew diagram is a horizontal strip (respectively, vertical strip) if each column (respectively, row) of the diagram contains exactly one box. Recall that given two partitions $\lambda$ and $\mu$, we say $\mu$ interlaces $\lambda$ if $\lambda_{i} \geq \mu_{i} \geq \lambda_{i-1}$ for all $i$. Then a skew diagram $\lambda / \mu$ is a horizontal strip (respectively, a vertical strip) if and only if $\mu$ interlaces $\lambda$ (respectively, $\mu^{\prime}$ interlaces $\lambda^{\prime}$ ).

Let $A$ be a set. A Young tableau with entries in $A$ is a Young diagram with an element of $A$ inserted in each box; we will call $A$ an alphabet, and an element of $A$ a letter. Let $\lambda$ be a partition and $\mu$ be an arbitrary sequence of non-negative integers indexed by $A$ such that $\sum_{a \in A} \mu_{a}=|\lambda|$. By a Young tableau of shape $\lambda$ and content $\mu$, we mean a Young tableau corresponding to partition $\lambda$ such that each letter $a \in A$ appears in $\mu_{a}$ boxes. When convenient, we will also use the notation $\mu=\left(a^{\mu_{a}}\right)_{a \in A}$.

2.2. Littlewood-Richardson coefficients. Let $\sigma$ and $\mu$ be partitions of length at most $m$. Given a third partition $\lambda$, let $c_{\sigma \mu}^{\lambda}$ be the Littlewood-Richardson coefficients. One way to interpret these non-negative integers is as multiplicities:

$$
L_{m}(\sigma) \otimes L_{m}(\mu) \cong \bigoplus_{\lambda} L_{m}(\lambda)^{\oplus c_{\sigma \mu}^{\lambda}} .
$$

Proposition 2.2.1. Let $\lambda, \mu$ and $\sigma$ be partitions. Then

(1) $c_{\sigma \mu}^{\lambda}=c_{\mu \sigma}^{\lambda}$ and $c_{\sigma \mu}^{\lambda}$ is nonzero only if $|\mu|+|\sigma|=|\lambda|$.

(2) $c_{\sigma^{\prime} \mu^{\prime}}^{\lambda^{\prime}}=c_{\sigma \mu}^{\lambda}$.

(3) Suppose further that $\mu=(n)$, a one row partition. Then $c_{\sigma(n)}^{\lambda}$ is either 1 or zero. Moreover, $c_{\sigma(n)}^{\lambda}=1$ if and only if the skew shape $\lambda / \sigma$ is a horizontal strip.

(4) Suppose further that $\mu=\left(1^{n}\right)$, a one column partition. Then $c_{\sigma\left(1^{n}\right)}^{\lambda}$ is either 1 or zero. Moreover, $c_{\sigma\left(1^{n}\right)}^{\lambda}=1$ if and only if the skew shape $\lambda^{\prime} / \sigma^{\prime}$ is a horizontal strip.

We refer the reader to [CW1, Appendix A] or [Sa for the details.

2.3. The Lie superalgebra $\mathfrak{g l}(m \mid n)$. The general linear Lie superalgebra $\mathfrak{g}=\mathfrak{g l}(m \mid n)$ is the vector superspace of $(m+n)$ by $(m+n)$ complex matrices, with the block-diagonal even subspace $\mathfrak{g}_{0}=\mathfrak{g l}(m) \oplus \mathfrak{g l}(n)$.

The standard Cartan subalgebra $\mathfrak{h}$ is the set of diagonal matrices in $\mathfrak{g l}(m \mid n)$. Let $h_{i}:=E_{i i} \in \mathfrak{h}$ be the elementary matrices with a 1 in the $(i, i)$ entry and 0 in all other entries, for $1 \leq i \leq m+n$. For $1 \leq i \leq m, 1 \leq j \leq n$, let $\delta_{i}, \epsilon_{j}$ be the elements in $\mathfrak{h}^{*}$ defined by

$$
\begin{gathered}
\delta_{i}\left(h_{k}\right)=\Delta_{i, k} \\
\epsilon_{j}\left(h_{l}\right)=\Delta_{j+m, l}
\end{gathered}
$$

where $\Delta_{x, y}=1$ if $x=y$, and 0 otherwise. The set $\left\{\delta_{i}, \epsilon_{j} \mid 1 \leq i \leq m, 1 \leq j \leq n\right\}$ forms a basis of $\mathfrak{h}^{*}$. 
The root system $\Phi$ of $\mathfrak{g}$ is the set

$$
\Phi=\left\{\delta_{k}-\delta_{l}, \epsilon_{i}-\epsilon_{j}, \delta_{k}-\epsilon_{i} \mid 1 \leq k, l \leq m, 1 \leq i, j \leq n\right\} .
$$

The Weyl group of $\mathfrak{g l}(m \mid n)$ is isomorphic to $S_{m} \times S_{n}$, where $S_{m}$ permutes the $\delta_{i}$ 's and $S_{n}$ permutes the $\epsilon_{j}$ 's. Note that not all simple systems are $W$-conjugate. In particular, there exist different choices of positive systems (and hence different choices of Borel subalgebras) that are not $W$-conjugate; see [CW1, Section 1.3].

2.4. Borel Subalgebras. By an $\epsilon-\delta$ sequence for $\mathfrak{g l}(m \mid n)$, we mean a sequence of $m$ (indistinguishable) $\delta$ 's and $n$ (indistinguishable) $\epsilon$ 's. For a given $\epsilon-\delta$ sequence $\underline{s}$, we can assign the indices $1, \ldots, m$ to the $\delta$ and the indices $1, \ldots, n$ to the $\epsilon$ by the increasing order in which they appear. Then $\Pi(\underline{s})$, the set of sequential differences of the indexed letters, forms a set of simple roots for $\Phi$. For example, $\Pi(\epsilon \delta \delta \epsilon)=\left\{\epsilon_{1}-\delta_{1}, \delta_{1}-\delta_{2}, \delta_{2}-\epsilon_{2}\right\}$.

It is easy to check that any simple system is $W$-conjugate to $\Pi(\underline{s})$ for some $\underline{s}$. Moreover, if $\underline{s}$ and $\underline{s}^{\prime}$ are distinct $\epsilon-\delta$ sequences, then $\Pi(\underline{s})$ and $\Pi\left(\underline{s}^{\prime}\right)$ are not conjugate, so these sequences index the simple systems of $\Phi$ up to conjugacy.

Let $\Phi^{+}(\underline{s})$ be the set of positive roots relative to $\Pi(\underline{s})$ for some sequence $\underline{s}$. Then we define the Borel subalgebra corresponding to $\underline{s}$ to be

$$
\mathfrak{b}(\underline{s})=\mathfrak{h} \oplus \bigoplus_{\alpha \in \Phi^{+}(\underline{s})} \mathfrak{g l}(m \mid n)_{\alpha} .
$$

Note that the even subalgebra $\mathfrak{b}(\underline{s})_{\overline{0}}$ is independent of $\underline{s}$; indeed, $\mathfrak{b}(\underline{s})_{\overline{0}}$ is exactly the standard Borel subalgebra of $\mathfrak{g l}(m \mid n)_{\overline{0}}=\mathfrak{g l}(m) \oplus \mathfrak{g l}(n)$.

Any Borel subalgebra is conjugate by inner automorphisms to $\mathfrak{b}(\underline{s})$ for some $\underline{s}$, and $\mathfrak{b}(\underline{s})$ is not conjugate to $\mathfrak{b}\left(\underline{s}^{\prime}\right)$ if $\underline{s} \neq \underline{s}^{\prime}$; therefore, for the remainder of this note a Borel subalgebra will mean $\mathfrak{b}(\underline{s})$ for some $\epsilon-\delta$ sequence $\underline{s}$, and we will abuse notation and identify the subalgebra with its corresponding sequence. For example, the standard Borel subalgebra of upper triangular matrices in $\mathfrak{g l}(m \mid n)$ is represented by the sequence

$$
\mathfrak{b}^{\text {st }}=\overbrace{\delta \ldots \delta}^{m} \overbrace{\epsilon \ldots \epsilon}^{n} ;
$$

2.5. Polynomial weights and hook partitions. Let $\omega=\sum_{i=1}^{m} \mu_{i} \delta_{i}+\sum_{j=1}^{n} \nu_{j} \epsilon_{j} \in \mathfrak{h}^{*}$. We call $\omega$ a polynomial weight if $\mu_{i}$ and $\nu_{j}$ are non-negative integers for all $i, j$.

Fix a Borel subalgebra $\mathfrak{b}$. Then each $(m \mid n)$-hook partition $\lambda$ corresponds to a polynomial weight as follows. For $1 \leq i \leq m$ and $1 \leq j \leq n$, let $d_{i}$ (resp. $e_{j}$ ) be the number of $\epsilon^{\prime}$ 's (resp. $\delta$ 's) appearing before the $i^{\text {th }} \delta$ (resp. $j^{\text {th }} \epsilon$ ) in $\mathfrak{b}$. For example, if $\mathfrak{b}=\delta \delta \epsilon \delta \epsilon \delta$, then

$$
d_{1}=d_{2}=0, d_{3}=1, d_{4}=2, e_{1}=2, e_{2}=3 .
$$

For $1 \leq i \leq m$ and $1 \leq j \leq n$, let $p_{i}=\max \left\{\lambda_{i}-d_{i}, 0\right\}$ and $q_{j}=\max \left\{\lambda_{j}^{\prime}-e_{j}, 0\right\}$. The pair $\left(p_{i}, q_{j}\right)$ is called the $\mathfrak{b}$-Frobenius coordinates; cf. [CW1. Then set

$$
\lambda^{\mathfrak{b}}=\sum_{i=1}^{m} p_{i} \delta_{i}+\sum_{j=1}^{n} q_{j} \epsilon_{j} .
$$

When $\mathfrak{b}=\mathfrak{b}^{\text {st }}$, there is a simpler description. Define the partitions $\mu=\left(\lambda_{1}, \ldots, \lambda_{m}\right)$ and $\sigma=\left(\lambda_{m+1}, \lambda_{m+2}, \ldots\right)^{\prime}$. Then we define $\lambda^{\natural}=\lambda^{\mathfrak{b}^{\mathrm{st}}}=\sum_{i=1}^{m} \mu_{i} \delta_{i}+\sum_{j=1}^{n} \sigma_{j} \epsilon_{j}$. 
2.6. Polynomial modules of $\mathfrak{g l}(m \mid n)$. An $\mathfrak{h}$-semisimple module $M$ is a polynomial module if the weights of $M$ are polynomial. In this note, by polynomial module we always mean a finite-dimensional polynomial module.

Given a weight $\omega$ and a Borel $\mathfrak{b}$, let $L(\mathfrak{b}, \omega)$ be the simple module of highest weight $\omega$ with respect to $\mathfrak{b}$. When $\mathfrak{b}=\mathfrak{b}^{\text {st }}$, we use the shorthand $L(\omega)=L\left(\mathfrak{b}^{\text {st }}, \omega\right)$. Then we have the following result (cf. [K, CK, CLW, CW1]).

Proposition 2.6.1. Fix a Borel subalgebra $\mathfrak{b}$.

(1) $\left\{L_{m \mid n}\left(\mathfrak{b}, \lambda^{\mathfrak{b}}\right): \lambda \in P_{m \mid n}\right\}$ is a complete list of pairwise non-isomorphic (finitedimensional) simple polynomial $\mathfrak{g l}(m \mid n)$-modules.

(2) $L_{m \mid n}\left(\mathfrak{b}, \lambda^{\mathfrak{b}}\right) \cong L_{m \mid n}\left(\lambda^{\natural}\right)$.

(3) The category of polynomial modules of $\mathfrak{g l}(m \mid n)$ is a semisimple tensor category.

\section{Branching RULE FOR $\mathfrak{g l}(m \mid n)$}

3.1. Branching Rule. Fix a Borel subalgebra $\mathfrak{b}$. Let $\hat{\mathfrak{b}}$ be the $\epsilon-\delta$ sequence obtained from $\mathfrak{b}$ by deleting the last entry in the sequence $\mathfrak{b}$, and $\hat{m}$ (respectively, $\hat{n}$ ) be the number of $\delta$ 's (respectively, $\epsilon$ 's) in $\hat{\mathfrak{b}}$. Also set $\hat{h}=h_{m} \in \mathfrak{h}$ if $\hat{n}=n$ and $\hat{h}=h_{m+n}$ if $\hat{m}=m$. Then we have a natural embedding

$$
\mathfrak{g l}(\hat{m} \mid \hat{n}) \cong \hat{\mathfrak{h}} \oplus \bigoplus_{\alpha \in \Phi \cap \hat{\mathfrak{h}}^{*}} \mathfrak{g l}(m \mid n)_{\alpha}
$$

where $\hat{\mathfrak{h}} \oplus \mathbb{C} \hat{h}=\mathfrak{h}$. With respect to this embedding, we have the following branching rule for an arbitrary Borel subalgebra.

Theorem 3.1.1 (Branching Rule for $\mathfrak{g l}(m \mid n)$ with respect to a general Borel). Let $\lambda$ be an $(m \mid n)$-hook partition and $\mathfrak{b}$ be a choice of Borel subalgebra for $\mathfrak{g l}(m \mid n)$. In the above notation,

$$
L_{m \mid n}\left(\mathfrak{b}, \lambda^{\mathfrak{b}}\right) \downarrow_{\mathfrak{g l}(\hat{m}, \hat{n})} \cong \bigoplus_{\sigma} L_{\hat{m} \mid \hat{n}}\left(\hat{\mathfrak{b}}, \sigma^{\hat{\mathfrak{b}}}\right)
$$

where the sum is taken over all $(\hat{m} \mid \hat{n})$-hook partitions $\sigma$ such that

(1) $\sigma^{\prime}$ interlaces $\lambda^{\prime}$ if $m=\hat{m}$,

(2) $\sigma$ interlaces $\lambda$ if $n=\hat{n}$.

Proof. First recall the statement of super Howe duality: as $\mathfrak{g l}(m \mid n) \times \mathfrak{g l}(k)$-modules,

$$
S\left(\mathbb{C}^{m \mid n} \otimes \mathbb{C}^{k}\right) \cong \bigoplus_{\substack{l(\lambda) \leq k \\ \lambda_{m+1} \leq n}} L_{m \mid n}\left(\mathfrak{b}, \lambda^{\mathfrak{b}}\right) \otimes L_{k}(\lambda) .
$$

Let $\mathfrak{p}=\mathfrak{g l}(\hat{m} \mid \hat{n}) \times \mathfrak{g l}(m-\hat{m} \mid n-\hat{n})$. Restricting (3.1) to a $\mathfrak{p} \times \mathfrak{g l}(k)$-module yields the decomposition

$$
S\left(\mathbb{C}^{m \mid n} \otimes \mathbb{C}^{k}\right) \downarrow_{\mathfrak{p} \times \mathfrak{g l}(k)} \cong \bigoplus_{\substack{l(\lambda) \leq k \\ \lambda_{m+1} \leq n}} L_{m \mid n}\left(\mathfrak{b}, \lambda^{\mathfrak{b}}\right) \otimes L_{k}(\lambda) .
$$

On the other hand, evidently $S\left(\mathbb{C}^{m \mid n} \otimes \mathbb{C}^{k}\right) \downarrow_{\mathfrak{p} \times \mathfrak{g l}(k)} \cong S\left(\mathbb{C}^{\hat{m} \mid \hat{n}} \otimes \mathbb{C}^{k}\right) \otimes S\left(\mathbb{C}^{m-\hat{m} \mid n-\hat{n}} \otimes \mathbb{C}^{k}\right)$. By applying Super Howe duality again to each tensor factor, we see that

$$
S\left(\mathbb{C}^{m \mid n} \otimes \mathbb{C}^{k}\right) \downarrow_{\mathfrak{p} \times \mathfrak{g l}(k)} \cong \bigoplus_{\sigma, \mu} L_{\hat{m} \mid \hat{n}}\left(\hat{\mathfrak{b}}, \sigma^{\hat{\mathfrak{b}}}\right) \otimes L_{k}(\sigma) \otimes L_{m-\hat{m} \mid n-\hat{n}}\left(\theta, \mu^{\theta}\right) \otimes L_{k}(\mu),
$$


where the sum is taken over all $\sigma$ and $\mu$ such that $\sigma$ is an $(\hat{m} \mid \hat{n})$-hook partition with $l(\sigma) \leq k$ and $\mu$ is a $(m-\hat{m} \mid n-\hat{n})$-hook partition with $l(\mu) \leq k$, and the notation $\theta=\delta$ (resp. $\epsilon)$ if $\hat{n}=n($ resp. $\hat{m}=m)$. In particular, since $\mathfrak{g l}(m-\hat{m} \mid n-\hat{n}) \cong \mathbb{C}, L_{m-\hat{m} \mid n-\hat{n}}\left(\theta, \mu^{\theta}\right)$ is 1-dimensional and thus

$$
S\left(\mathbb{C}^{m \mid n} \otimes \mathbb{C}^{k}\right) \downarrow_{\mathfrak{g l} l(\hat{m} \mid \hat{n}) \times \mathfrak{g l}(k)}=\bigoplus_{\sigma, \mu} L_{\hat{m} \mid \hat{n}}\left(\hat{\mathfrak{b}}, \sigma^{\hat{\mathfrak{b}}}\right) \otimes\left(L_{k}(\sigma) \otimes L_{k}(\mu)\right) .
$$

Then using (2.1),

$$
\begin{aligned}
S\left(\mathbb{C}^{m \mid n} \otimes \mathbb{C}^{k}\right) \downarrow_{\mathfrak{g l} l}(\hat{m} \mid \hat{n}) \times \mathfrak{g l}(k) & =\bigoplus_{\sigma, \mu} L_{\hat{m} \mid \hat{n}}\left(\hat{\mathfrak{b}}, \sigma^{\hat{\mathfrak{b}}}\right) \otimes \bigoplus_{\lambda} L_{k}(\lambda)^{\oplus c_{\sigma \mu}^{\lambda}} \\
& =\bigoplus_{\sigma, \mu, \lambda} L_{\hat{m} \mid \hat{n}}\left(\hat{\mathfrak{b}}, \sigma^{\hat{\mathfrak{b}}}\right) \otimes L_{k}(\lambda)^{\oplus c_{\sigma \mu}^{\lambda}}
\end{aligned}
$$

Comparing (3.2) and (3.4), we obtain

$$
L_{m \mid n}\left(\mathfrak{b}, \lambda^{\mathfrak{b}}\right) \downarrow_{\mathfrak{g l} l(\hat{m} \mid \hat{n})}=\bigoplus_{\sigma, \mu} L_{\hat{m} \mid \hat{n}}\left(\hat{\mathfrak{b}}, \sigma^{\hat{\mathfrak{b}}}\right)^{\oplus c_{\sigma \mu}^{\lambda}}
$$

Since $\{m-\hat{m}, n-\hat{n}\}=\{0,1\}, \mu$ is either a row or column partition. The result follows by Proposition 2.2.1.

Remark 3.1.2. The case $\mathfrak{b}=\mathfrak{b}^{\text {st }}$ had been proved in $[\mathrm{BR}]$ using the commuting actions of $\mathfrak{g l}(m \mid n)$ and $S_{d}$ on $\left(\mathbb{C}^{(m \mid n)}\right)^{\otimes d}$.

3.2. The Gelfand-Tsetlin basis and $\mathfrak{b}$-semistandard tableaux. By iterating Theorem 3.1.1, one obtains a linear basis of $L_{m \mid n}\left(\mathfrak{b}, \lambda^{\mathfrak{b}}\right)$. These basis vectors are in bijection with the sequences of triples $\left(m_{k}, n_{k}, \lambda^{k}\right)$ where $m_{k}, n_{k}$ are non-negative integers and $\lambda^{k}$ are partitions satisfying the following conditions.

(1) Either $m_{k}=m_{k+1}$ and $n_{k}=n_{k+1}+1$ or $m_{k}=m_{k+1}+1$ and $n_{k}=n_{k+1}$.

(2) $\lambda^{k}$ is an $\left(m_{k} \mid n_{k}\right)$-hook partition.

(3) $\lambda^{k} / \lambda^{k+1}$ is a vertical strip if $m_{k}=m_{k+1}$ or a horizontal strip otherwise (or the empty diagram).

These sequences may be realized in terms of certain tableaux of $\lambda$. Let $A$ denote the alphabet set $\{1, \ldots, m, \overline{1}, \ldots, \bar{n}\}$ and $\mathfrak{b}$ be the following $\epsilon-\delta$ sequence:

$$
\mathfrak{b}=\overbrace{\delta \delta \ldots \delta}^{\mu_{1}} \overbrace{\epsilon \epsilon \epsilon}^{\nu_{1}} \ldots \ldots \overbrace{\delta \ldots \delta}^{\mu_{t}} \overbrace{\epsilon \ldots \epsilon}^{\nu_{t}},
$$

where $\mu_{1}$ and $\nu_{t}$ are non-negative and all other $\mu_{i}, \nu_{j}$ are positive integers. For convenience, set $\nu_{0}=0$. Define the total order $<_{\mathfrak{b}}$ on $A$ by setting

$$
\begin{gathered}
i<_{\mathfrak{b}} j \text { if } 1 \leq i<j \leq m, \quad \bar{k}<_{\mathfrak{b}} \bar{l} \text { if } 1 \leq k<l \leq n, \\
i<_{\mathfrak{b}} \bar{k} \text { if } i \leq \sum_{g=1}^{r} \mu_{g} \text { and } k>\sum_{g=0}^{r-1} \nu_{g} \text { for some } 1 \leq r \leq t, \\
\bar{k}<_{\mathfrak{b}} i \text { if } i>\sum_{g=1}^{r} \mu_{g} \text { and } k \leq \sum_{g=0}^{r} \nu_{g} \text { for some } 1 \leq r \leq t .
\end{gathered}
$$

A tableau in $A$ will be called $\mathfrak{b}$-semistandard if the following three conditions are satisfied:

(1) The entries are weakly increasing along each row and column with respect to $<_{\mathfrak{b}}$,

(2) The entries from $\{1,2, \ldots, m\}$ are strictly increasing along each column,

(3) The entries from $\{\overline{1}, \overline{2}, \ldots, \bar{n}\}$ are strictly increasing along each row. 
The combinatorics of such tableaux have been studied in some detail, and in particular it is known that there is a content-preserving bijection between $\mathfrak{b}$-semistandard tableaux and $\mathfrak{b}^{\text {st }}$-semistandard tableaux; cf. $\mathrm{Kw}$.

Proposition 3.2.1. The module $L_{m \mid n}\left(\mathfrak{b}, \lambda^{\mathfrak{b}}\right)$ has a basis indexed by $\mathfrak{b}$-semistandard tableaux of shape $\lambda$ which is obtained by iterated application of the branching rule. The entries determine which simple summand the basis vector belongs to at each application of the branching rule.

Proof. Order $A$ from largest to smallest according to $\mathfrak{b}$ to obtain $A=\left\{a_{1}>_{\mathfrak{b}} \ldots>_{\mathfrak{b}} a_{n+m}\right\}$. Then given a sequence $\left(m_{k}, n_{k}, \lambda^{k}\right)$ associated to a linear basis element we can define a unique $\mathfrak{b}$-semistandard tableau: give each box in $\lambda^{k} / \lambda^{k+1}$ the entry $a_{k}$. Clearly the resulting tableau is uniquely determined by the sequence of partitions. On the other hand, given a $\mathfrak{b}$-semistandard tableau it is evident that stripping away the boxes with entry $a_{1}$, then those with $a_{2}$, etc. defines a sequence of partitions corresponding to a basis element.

Example 3.2.2. Consider $\mathfrak{g l}(3 \mid 2)$ and $\mathfrak{b}=\delta \epsilon \epsilon \delta \delta$. We choose the following partitions $\lambda=\lambda^{1}=(4,3,3,2,2,1), \lambda^{2}=(3,3,2,2,2), \lambda^{3}=(3,2,2,2), \lambda^{4}=(2,1,1,1), \lambda^{5}=(1)$.

In this case, the ordered alphabet is

$$
1<_{\mathfrak{b}} \overline{1}<_{\mathfrak{b}} \overline{2}<_{\mathfrak{b}} 2<_{\mathfrak{b}} 3 .
$$

Following the algorithm above, we find the corresponding $\mathfrak{b}$-semistandard tableau to be

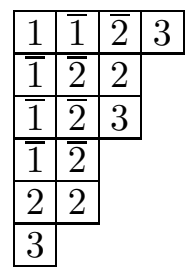

We note that if $\mu$ is the content of a $\mathfrak{b}$-semistandard tableau $T$ and we write

$$
\mu=\left(1^{\mu_{1}}, 2^{\mu_{2}}, \ldots, m^{\mu_{m}}, \overline{1}^{\mu_{\overline{1}}}, \ldots, \bar{n}^{\mu_{\bar{n}}}\right)
$$

then the basis vector corresponding to $T$ has weight

$$
\mu=\sum_{i=1}^{m} \mu_{i} \delta_{i}+\sum_{j=1}^{n} \mu_{j} \epsilon_{j} .
$$

3.3. An analogue of the Kostka numbers. The branching rule implies that the number of $\mathfrak{b}$-semistandard tableaux of shape $\lambda$ is equal to the dimension of $\mathfrak{g l}(m \mid n)$-module $L_{m \mid n}\left(\mathfrak{b}, \lambda^{\mathfrak{b}}\right)$, which is the same module as $L_{m \mid n}\left(\lambda^{\mathfrak{\natural}}\right)$ by Proposition 2.6.1. Comparing the Gelfand-Tsetlin bases, we obtain the following result, which is a special case of $\mathrm{Kw}$, Proposition 2.11].

Corollary 3.3.1. Fix an $(m \mid n)$-hook partition $\lambda$ and a content $\mu$. The number $K_{\lambda, \mu}^{\mathfrak{b}}$ of $\mathfrak{b}$-semistandard tableaux of shape $\lambda$ and content $\mu$ is independent of the choice of $\mathfrak{b}$. In particular, there is a well-defined number $K_{\lambda, \mu}^{(m \mid n)}=K_{\lambda, \mu}^{\mathfrak{b}}$ which is an analogue of the Kostka number $K_{\lambda, \mu}$.

Example 3.3.2. Consider $\mathfrak{g l}(2 \mid 1)$ and $\lambda=(3,2,1)$

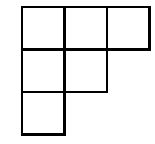


Consider two different $\epsilon-\delta$ sequences $\mathfrak{b}_{1}=\mathfrak{b}^{\text {st }}=\delta \delta \epsilon$ and $\mathfrak{b}_{2}=\delta \epsilon \delta$. One may check that all possible $\mathfrak{b}_{1}$-semistandard tableaux of shape $\lambda$ are

\begin{tabular}{|c|c|c|c|c|c|c|c|c|c|}
\hline 1 & \begin{tabular}{l|l}
1 & 1 \\
\end{tabular} & 1 & 1 & \begin{tabular}{l|l}
1 & 1 \\
\end{tabular} & 1 & & 2 & \begin{tabular}{|l|}
1 \\
\end{tabular} & $1 \overline{1}$ \\
\hline 2 & 2 & & 2 & $\begin{array}{ll}1 \\
1\end{array}$ & 2 & 2 & & \begin{tabular}{|l|}
2 \\
\end{tabular} & \\
\hline$\overline{1}$ & & & $\overline{1}$ & & $\overline{1}$ & & & \begin{tabular}{|l|}
1 \\
\end{tabular} & \\
\hline 1 & \begin{tabular}{l|l}
1 & 2 \\
\end{tabular} & 2 & 1 & \begin{tabular}{l|l}
1 & $\overline{1}$ \\
\end{tabular} & 1 & 2 & 2 & \begin{tabular}{|l|}
1 \\
\end{tabular} & $2 \overline{1}$ \\
\hline$\frac{2}{7}$ & $\overline{1}$ & & $\frac{2}{2}$ & 2 & $\frac{2}{7}$ & 1 & & \begin{tabular}{|l|}
2 \\
7
\end{tabular} & \\
\hline$\overline{1}$ & & & $\overline{\overline{1}}$ & & $\overline{\mid \overline{1}}$ & & & \begin{tabular}{|l|}
$\overline{1}$ \\
\end{tabular} & \\
\hline
\end{tabular}

and all possible $\mathfrak{b}_{2}$-semistandard tableaux are

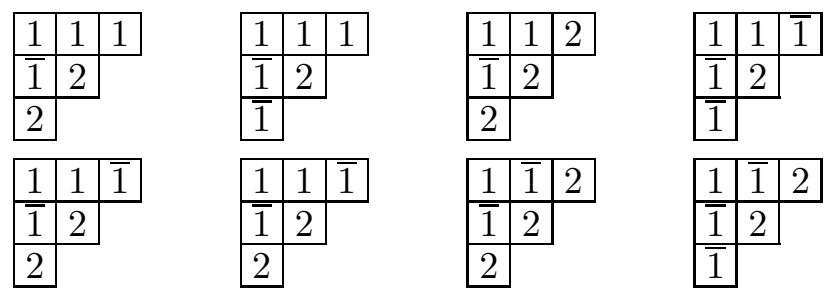

Hence the number of semistandard tableaux are the same.

In both cases, there are two semistandard tableaux with content $\left(1^{2}, 2^{2} \mid \overline{1}^{2}\right)$ and there is only one semistandard tableau for other possible contents.

\section{REFERENCES}

[BR] A. Berele and A. Regev, Hook Young Diagrams with Applications to Combinatorics and to Representations of Lie Superalgebras, Adv. Math. 64 (1987), 118-175.

[CK] S.-J. Cheng and J.-H. Kwon, Howe duality and Kostant homology formula for infinite-dimensional Lie superalgebras, Int. Math. Res. Not. 2008, Art. ID rnn 085, 52 pp.

[CW1] S.-J. Cheng and W. Wang, Dualities and Representations of Lie Superalgebras, Graduate Studies in Mathematics 144, AMS, 2013.

[CW2] S.-J. Cheng and W. Wang, Howe duality for Lie superalgebras, Compositio Math. 128 (2001), 55-94.

[CLW] S.-J. Cheng, N. Lam and W. Wang, Super duality and irreducible characters of ortho-symplectic Lie superalgebras, Inventiones Mathematicae 183 (2011), 189-224.

[GW] R. Goodman and N. Wallach, Symmetry, Representations, and Invariants, GTM 255, Springer, New York, 2009.

[K] V. Kac, Lie superalgebras, Adv. Math. 26 (1977), 8-96.

[Kw] J. H. Kwon, Rational semistandard tableaux and character formula for the Lie superalgebra $\mathfrak{g l}(\infty \mid \infty)$, Adv. Math. 217 (2008), 713-739.

[Mo] A. Molev, Gelfand-Tsetlin bases for classical Lie algebras, in "Handbook of Algebra", Vol. 4, (M. Hazewinkel, Ed.), Elsevier, 2006, 109-170.

[Sa] B. Sagan, The Symmetric Group: Representations, Combinatorial Algorithms, and Symmetric Functions, 2nd edition, GTM 203, Springer-Verlag, New York, 2001.

[Se1] A. Sergeev, An analog of the Classical Invariant Theory, I, II, Michigan J. Math. 49 (2001), $113-146,147-168$.

[Se2] A. Sergeev, The tensor algebra of the identity representation as a module over the Lie superalgebras $g l(m, n)$ and $Q(n)$, Math. USSR Sbornik 51 (1985), 419-427.

[SV] N. Stoilova and J. Van der Jeugt, Gelfand-Zetlin Basis and Clebsch-Gordan Coefficients for Covariant Representations of the Lie superalgebra $\mathfrak{g l}(m \mid n)$, J. Math. Phys, 51 (2010), art. no. 09352, $15 \mathrm{pp}$.

Department of Mathematics, University of Virginia, Charlottesville, VA 22904, USA

E-mail address: sic5ag@virginia.edu (Clark), st5tp@virginia.edu (Thamrongpairoj)

Institute of Mathematics, Academia Sinica, Taipei City 10617, Taiwan

E-mail address: ynp@math.sinica.edu.tw (Peng) 